\title{
Iterative Noise Reduction Algorithm-Based Cone Beam Computed Tomography Image Analysis for Dental Pulp Disease in Root Canal Therapies
}

\author{
Kai Zhang $(D)$ and Weidong Yang $(D)$ \\ Department of Endodontics, Nanjing Stomatological Hospital, Medical School of Nanjing University, 30 Zhongyang Road, \\ Nanjing, Jiangsu 210008, China \\ Correspondence should be addressed to Weidong Yang; 201703404@stu.ncwu.edu.cn
}

Received 13 December 2021; Revised 20 January 2022; Accepted 22 January 2022; Published 15 February 2022

Academic Editor: M Pallikonda Rajasekaran

Copyright (c) 2022 Kai Zhang and Weidong Yang. This is an open access article distributed under the Creative Commons Attribution License, which permits unrestricted use, distribution, and reproduction in any medium, provided the original work is properly cited.

\begin{abstract}
This study was aimed to explore the application value of cone beam computed tomography (CBCT) imaging technology based on iterative noise reduction (INR) algorithm in the evaluation of the efficacy of root canal therapy (RCT) for dental pulp disease. Eighty eight dental pulp patients who underwent RCT were taken as the research subjects, and INR algorithm-based CBCT and digital periapical film (DPF) were adopted for examination. Basic information of patients, image performance, and filling quality was recorded. It was found that the INR algorithm-based CBCT images achieved favorable noise reduction effects both within and between slices. Filling length of root canal was evaluated by CBCT and DPF, and differences between them were considerable $(P<0.05)$. Root canal closeness was evaluated by CBCT and DPF, and substantial differences between them were shown $(P<0.05)$. Moreover, differences on root canal filling quality examined by CBCT and DPF were also remarkable $(P<0.05)$. Applying CBCT imaging technology based on INR algorithm to the analysis of the curative effect of dental pulp patients can better retain the image information and can also more accurately evaluate the curative effect of different RCTs.
\end{abstract}

\section{Introduction}

Dental pulp disease is an important reason for patients to see a doctor, which causes the lack of teeth. At present, RCT is often utilized clinically for treatment [1-3]. Traditional $\mathrm{X}$-ray inspection has low cost, low radiation dose, and simple operation. DPF is an effective and commonly utilized root canal curative effect inspection method, which has the advantages of high image resolution $[4,5]$. However, with the continuous improvement of RCT technology, 2D X-ray inspection methods can no longer meet the needs in some complex dental diagnosis and treatment. The emergence of CT technology has solved this problem, but due to its poor resolution in jaws, teeth, and other tissues, CT imaging has limited application scope and cannot be utilized as a routine oral examination technology. CBCT adopts wide-beam scanning imaging, which can scan and detect from the three directions of the transverse plane, the sagittal plane, and the coronal plane. It can not only deal with distortion and overlap of $2 \mathrm{D}$ images but also has high tissue resolution and low-dose radiation, which is widely utilized in the fields of endodontics, oral, and maxillofacial surgery [6-8].

In medical CT equipment, there are three interaction modes between X-rays and the detection object, namely, photoelectric effect, Compton effect, and coherence effect. During the actual medical examination, the incident $X$ photons will be partially absorbed and scattered. Conventional CT is difficult to have scattering, while in CBCT, its $\mathrm{X}$-ray source is cone-shaped and is easily scattered between tomographic images, which affects the quality of the reconstructed image [9]. Therefore, how to improve the imaging quality of CBCT images has become a key research issue in this field. In recent studies, the penalty-weighted least squares noise reduction algorithm is utilized more in 
CBCT projection noise reduction [10]. The penalty-weighted least squares noise reduction algorithm can not only suppress the noise but also retain the edge of the image. The penalty-weighted least squares denoising algorithm is currently a research hotspot in CBCT, and many denoising algorithms are optimized based on the penalty-weighted least squares denoising algorithm $[11,12]$. DPF is a hard material used in the diagnosis of dental caries in digital imaging technology. The length of the captured tooth is greatly affected by the structure of the tooth. Therefore, its application in endodontic root canal treatment is not ideal.

Based on the above advantages, the penalty-weighted least squares noise reduction algorithm was fused with the fuzzy entropy mean filtering algorithm. Then, the CBCT imaging technology based on INR algorithm was proposed and applied to the curative effect evaluation of RCT for dental pulp disease. The inspection results of root canal filling length, root canal closeness, and root canal filling quality were compared between DPF and INR-based CBCT, to explore the application value of CBCT imaging technology based on INR algorithm in RCT for dental pulp disease, which aimed to provide a theoretical basis for the judgment of root canal filling effect and the application of CBCT in RCT.

\section{Materials and Methods}

2.1. Research Subjects. Eighty eight patients with dental pulp received RCT in hospital from October 2017 to October 2020 and were taken as the research subjects, including 48 males and 40 females and aged 18-70 years old, with an average age of $46.35 \pm 3.22$ (year old). All patients had a total of 110 teeth involved, with 28 anterior teeth, 24 upper premolars, 12 lower premolars, 20 upper molars, and 26 mandibular molars. There were 234 cases of root canal in total. This study had been approved by the ethics committee, and all patients signed the informed consent forms and agreed to be included.

Inclusion criteria: (I) patients with pulpitis or apical periodontitis; (II) patients received RCT; (III) patients received CBCT and DPF examination; (IV) examinations of various parts were conducted carefully and with complete process; (V) patients with complete clinical data. Exclusion criteria: (I) patients with other pulp diseases; (II) patients who did not receive RCT; (III) patients with unclear CBCT image.

2.2. Therapy Methods. All patients underwent RCT. Firstly, the tooth was taken with the initial tip film to understand and determine the shape, the number of root canal, and other related information, and then, the routine pulping, pulping removing, and other operations were performed. The root canal preparation was carried out by the step-by-step retreat method, and the k-shaped reaming file and reaming drill were utilized. At the same time, $3 \% \mathrm{H}_{2} \mathrm{O}_{2}$ and normal saline were adopted to continuously flush root canal. Root canal working length was determined using Root ZX electronic apex locator.
FC (Cresol and formaldehyde Solution) cotton was utilized to twist the dressing, and the number of patient visits would depend on the inflammation. When there was no exudation in the root canal of the affected tooth, the root filling operation would be started. The root canal of the affected tooth was filled with iodoform paste and 0.2 -taper gutta percha.

2.3. Imaging Test Methods. After the root canal filling was completed, imaging scans were performed, and the digital $\mathrm{X}$-ray camera and the CBCT scanner were utilized to carefully check all the patients' teeth. DPF inspection method: parallel projection technology was adopted, the exposure parameters were set to voltage $60 \mathrm{kv}$, current $8 \mathrm{~mA}$, scanning time within the range of $0.15-0.20 \mathrm{~s}$. The patient wore a lead collar during scanning, and the scanning data were transmitted to the instrument's own software for calculation and analysis. CBCT examination method: the patient stood in front of the instrument, and the patient needed to wear a lead collar and a lead suit when shooting. The patient's chin was placed in the support and fixed, and the orbital ears were kept parallel to the ground. The exposure parameters were set to voltage $90 \mathrm{kv}$, current $12 \mathrm{~mA}$, and scan time $12 \mathrm{~s}$, and scan data were transmitted to the instrument's own software for reconstruction and analysis. Professional image display was adopted for reading.

Two dentists with more than 5 years of clinical experience were chosen to read the reconstructed images to evaluate the length of root filling and closeness. The specific criteria were shown in Table 1.

2.4. CBCT Image Based on INR Algorithm. Noises in CBCT projection data are classified into Gaussian noise and impulse noise. First, the threshold judgment method is utilized to distinguish the two kinds of noises. The CBCT projection data can be expressed as follows after system calibration and logarithmic transformation:

$$
P_{c}=\left[P_{i, k}\right]_{m^{*} a}
$$

where $m$ is the number of detectors, $a$ is the number of rotation angles, $i$ is the detector channel, $k$ is the projection angle, and $P_{i, k}$ is the projection data value.

It is assumed that $a=\left|p_{i, k}-m_{r}\right|$ and $b=1 /$ count $-1 \operatorname{sum}\left(\operatorname{select}_{\text {count }}\left(\operatorname{sort}_{\mathrm{asc}}\left(\left|p_{r}-p_{i, k}\right|\right)\right)\right) . r$ is the radius of the neighborhood, $m_{r}$ is the mean value of the neighborhood window, asc is the monotonous increasing order of neighborhood pixels, and cont is the number of pixels, which is generally 3 . The noise threshold is judged as follows. (I) impulse noise pollution, $a \geq T_{1}$ and $b \geq T_{2}$. (II) if condition I is not satisfied, it is Gaussian noise pollution. The threshold $T_{1}$ is determined by the mean variance of the neighborhood window.

Then, the fuzzy entropy weighted mean (FEWM) filtering algorithm is utilized to remove impulse noise, and the membership function expressions constructed are as follows: 
TABLE 1: Judgment criteria for different images.

\begin{tabular}{|c|c|c|}
\hline Test method & Test result & Judgment criteria \\
\hline DPF & $\begin{array}{l}\text { Well filled } \\
\text { Over filled } \\
\text { Lacked filled }\end{array}$ & $\begin{array}{c}\text { The distance between the root filling material and the radiological root tip is within } 0-2 \mathrm{~mm} \\
\text { Root filling material }>\text { radiological root tip } \\
\text { The distance between the root filling material and the radiological root tip }>2 \mathrm{~mm}\end{array}$ \\
\hline СBCT & $\begin{array}{l}\text { Well filled } \\
\text { Over filled } \\
\text { Lacked filled }\end{array}$ & $\begin{array}{l}\text { Root filling material stops at the root canal end. } \\
\text { Root filling material }>\text { root canal end } \\
\text { Root filling material }<\text { root canal end }\end{array}$ \\
\hline
\end{tabular}

$$
\begin{aligned}
\widehat{\mu}\left(p_{i}\right) & =\frac{\bar{P}_{c, r}}{\max \left(\bar{P}_{c, r}\right)}, \\
\bar{P}_{c, r} & =\left[P_{i, k}\right]_{\left(2 r_{i}+1\right) *\left(2 r_{i}+1\right)} .
\end{aligned}
$$

To avoid over-smoothing the image quality, equation (2) can be expressed as follows:

$$
\widehat{E}\left(\widehat{\mu}\left(P_{i}\right)\right)=\frac{1}{2}\left(\widehat{\mu}\left(P_{i}\right) * \exp \left(1-\widehat{\mu}\left(P_{i}\right)\right)+\left(1-\widehat{\mu}\left(P_{i}\right)\right) \exp \left(\widehat{\mu}\left(P_{i}\right)\right) .\right.
$$

After a maximum value and a minimum value are removed from the obtained fuzzy entropy, the estimated value of $\bar{P}_{c, r}$ is calculated according to the following equation:

$$
\widetilde{\bar{P}}_{c, r}=\frac{1}{\operatorname{sum}(E)} \sum_{j \in\left(2 r_{i}+1\right) *\left(2 r_{i}+1\right)}\left(E_{j} * \bar{P}_{c, j}\right) .
$$

Penalized weighted least squares algorithm (PWLS) is utilized to remove Gaussian noise, and the approximate FDK algorithm is utilized to reconstruct the projection image. Since slices have isotropic characteristics, CBCT projection data are prone to scatter, which affect the quality of the reconstructed image, such as noise interference, many scattering artifacts, and low contrast.

The main steps of the PWLS-FEWM-FL algorithm are initializing any slice projection data, calculating the noise variance of the projection data, and setting the threshold and the number of iterations of the edge enhancement operator. Then, iterative (PWLS) noise reduction processing is performed on any pixel. $3 \times 3$ neighborhood window is chosen, and after the threshold is judged, if it is impulse noise, FEWM will be adopted to filter it; if it is Gaussian noise, equations (2) and (4) will be adopted to calculate it, which is substituted into the following equation to complete a pixel iteration:

$$
y_{i}^{(n+1)}=\frac{p_{i}+\beta \sigma_{i}^{2}\left(\sum_{m \in V_{i}^{1}} \omega_{i m} y_{m}^{(n+1)}+\sum_{m \in V_{i}^{2}} \omega_{i m} y_{m}^{(n)}\right)}{1+\beta \sigma_{i}^{2} \sum_{m \in V_{i}} \omega_{i m}},
$$

where $n$ is the number of iterations, $i$ is the center pixel, $V_{i}^{1}$ is the neighborhood on the left and top of the $i$ pixel, and $V_{i}^{2}$ is the neighborhood on the right and bottom of the $i$ pixel. If the above equation converges to a relatively stable solution of a certain standard metric, the iteration stops.

The intraslice and interslice spatial resolution of CBCT projection data are the same. A constrained optimization 3D model of penalty-weighted least squares algorithm (TPWLS) was established to reduce noise between slices:

$$
\widehat{Y}_{3}=\arg \min _{Y_{3} \geq 0}\left(W\left\|Y_{3}-P_{3}\right\|_{2}^{2}+\beta R\left(Y_{3}\right)\right) \text {, }
$$

where $\beta$ is the regularization parameter, $Y_{3}$ is the ideal CBCT projection data, $P_{3}$ is the result of the actual projection data processing, and $R(\bullet)$ is the regularization term, and the expression is as follows:

$$
R\left(Y_{i, j, a}\right)=\sum_{i, j, a \in[-r, r]} \omega_{i, j, a} *\left(\left(Y_{i, j+r, a}-Y_{i, j, a}\right)^{2}+\left(Y_{i, j+r, a}-Y_{i, j, a}\right)^{2}+\left(Y_{i, j+r, a}-Y_{i, j, a}\right)\right)^{2}
$$

where $w$ is the weight and $r$ is the radius of the neighborhood.

The algorithm flowchart proposed in this work is shown in Figure 1.
2.5. Image Quality Evaluation Method of CBCT Based on INR Algorithm. Three CT projection data slices, slice 1, slice 2, and slice 3, are randomly selected, and the image quality is evaluated using the peak signal-to-noise ratio (PSNR) and 


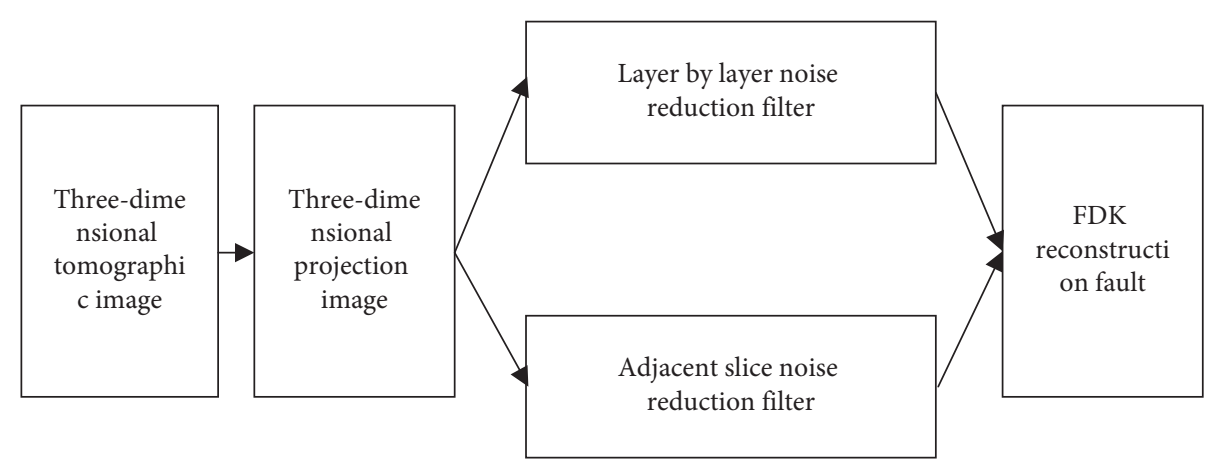

FIgURE 1: CBCT image based on INR algorithm.

correlation coefficient (CORR). The larger the CORR value, the closer the reconstructed image to the original image and the better the ability to retain detailed structure. The larger the PSNR value, the stronger the image noise reduction ability. The specific expressions of PSNR and CORR are as follows:

$$
\begin{aligned}
& \operatorname{CORR}=\frac{\sum_{i=1}^{N} \sum_{j=1}^{M}\left(f(i, j)-m_{f}\right)\left(\hat{f}(i, j)-m_{\hat{f}}\right)}{\sqrt{\sum_{i=1}^{N} \sum_{j=1}^{M}\left(f(i, j)-m_{f}\right)\left(\hat{f}(i, j)-m_{\hat{f}}\right)^{2}}}, \\
& \text { PSNR }=10 \times \log \left(\frac{M * N * H_{\max }^{2}}{\sum_{i=1}^{N} \sum_{j=1}^{M}(\widehat{f}(i, j)-f(i, j))}\right) .
\end{aligned}
$$

In the above equations, $f$ is the original image, $\widehat{f}$ is the reconstructed image, $m$ is the mean gray value, $H_{\max }$ is the maximum gray value, $M \times N$ is the image size, $\widehat{f}(i, j)$ is the gray value of the reconstructed image at $(i, j)$, and $f(i, j)$ is the gray value of the original image at $(i, j)$.

2.6. Statistical Methods. SPSS20.0 statistical software was employed to analyze the indicators obtained by the algorithm. The chi-square test was adopted to evaluate and analyze the results of algorithm indicator of CBCT and DPF.

\section{Results}

3.1. Image Quality Evaluation Result of CBCT Based on INR Algorithm. For the intraslice noise reduction algorithm, the PSNR (peak signal-to-noise ratio) and CORR (correlation coefficient) values of the algorithm proposed in this work were superior to those of the PWLS algorithm, as shown in Figures 2 and 3. For the interslice noise reduction algorithm, the PSNR and CORR values of the proposed algorithm were better than those of the TPWLS algorithm, as shown in Figures 4 and 5. Based on the above results, the CBCT image of the INR algorithm proposed had achieved favorable noise reduction effects within and between slices, and the detailed information was maintained well.

3.2. Imaging Characteristics of Some Patients. CBCT imaging technology and DPF were utilized to evaluate the efficacy of RCT in patients with dental pulp disease. The CBCT imaging results of some patients are shown in Figure 6. Image of patient $a$ appeared to be lacked filled, and the gap can be observed from the distance. The image of patient $b$ is manifested as well filled, and the root canal was opened on the cheek side of the root. The image of patient $c$ is manifested as over filled, and there was no gap. The DPF examination results of some patients are shown in Figure 7. The image of patient $a$ shows that it was well filled and no gap existed. The image of patient $b$ is manifested as lacked filled and that of patient $c$ was well filled and was with gap.

3.3. Evaluation and Comparison Results of Root Canal Length. $\mathrm{CBCT}$ imaging and DPF were utilized to evaluate the filling length of root canal after RCT, and the results are shown in Figure 8. INR algorithm-based CBCT imaging technology was adopted to evaluate the length of root canal, and it turned out that 132 patients were displayed as well filled, 26 patients were displayed as over filled, and 76 patients were displayed as lacked filled. When DPF was adopted to evaluate the length of root canal, 169 patients were displayed as well filled, 19 patients were displayed as over filled, and 46 patients were displayed as lacked filled. CBCT imaging technology and DPF were utilized to evaluate the filling length of root canal, and differences between the results were very substantial $(P<0.05)$.

3.4. Evaluation and Comparison Results of Root Canal Closeness. CBCT and DPF were adopted to evaluate root canal closeness after RCT, and the results are shown in Figure 9. INR algorithm was utilized to improve the CBCT imaging technology for root canal closeness evaluation, and 139 patients showed no gap and 95 patients showed gap. When DPF was adopted for root canal closeness evaluation, 196 patients showed no gap and 38 patients showed gap. After CBCT imaging technology and DPF were utilized to evaluate root canal closeness, differences between the results were highly notable $(P<0.05)$.

\subsection{Evaluation and Comparison Results of Root Canal Filling} Quality. For the RCT filling, well filled and no gap was defined as satisfactory, and the rest were unsatisfactory. The INR algorithm was utilized to improve the CBCT images, which was adopted to evaluate the root canal filling quality and was compared with that of DPF. The results are shown 


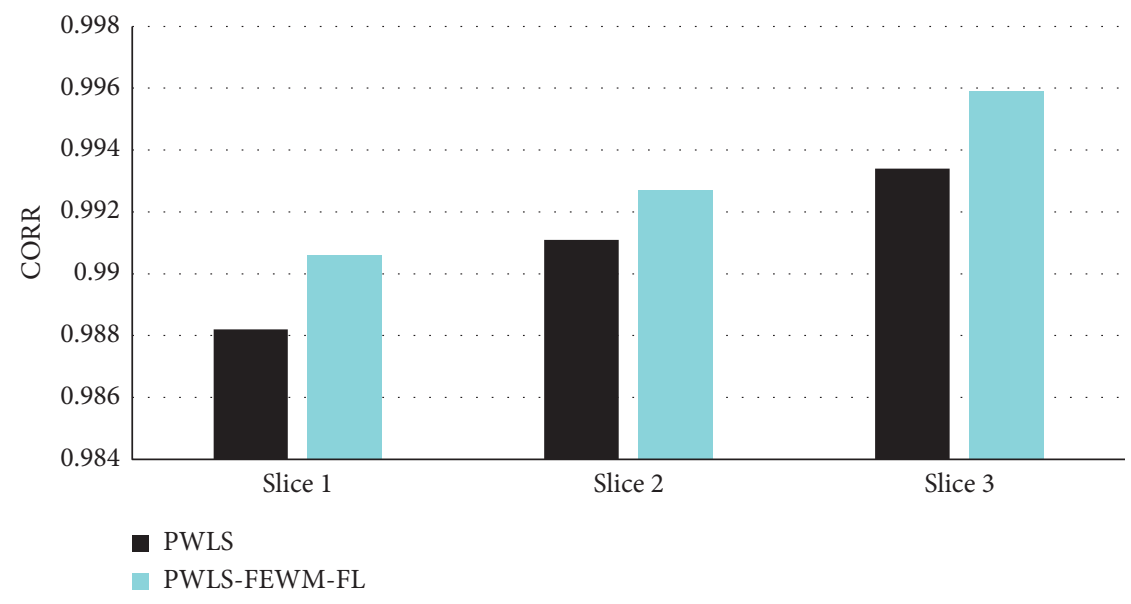

Figure 2: Contrast of the CORR value of noise reduction algorithm within slices.

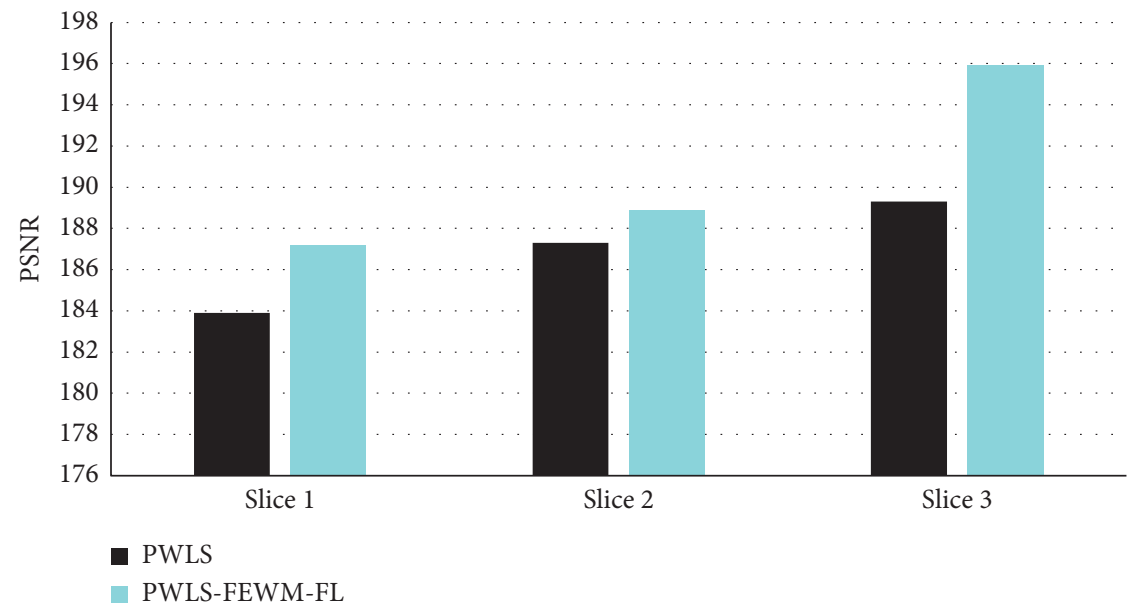

FIGURE 3: Contrast of the PSNR value of noise reduction algorithm within slices.

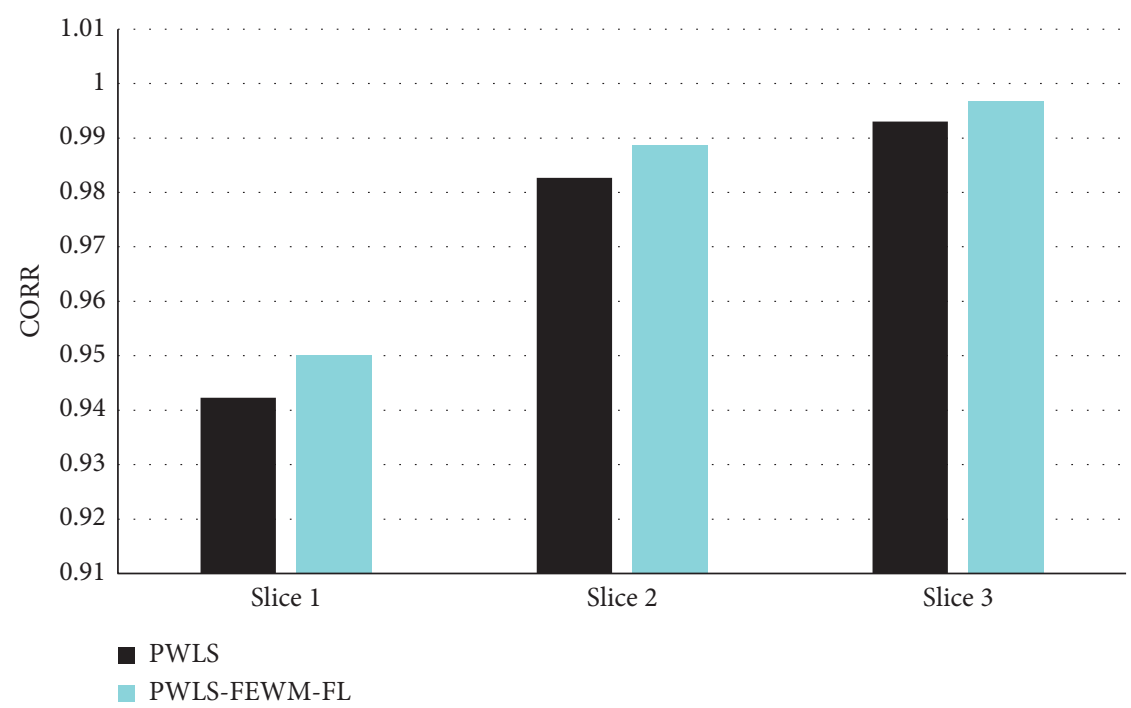

FIGURE 4: Contrast of the CORR value of noise reduction algorithm between slices. 


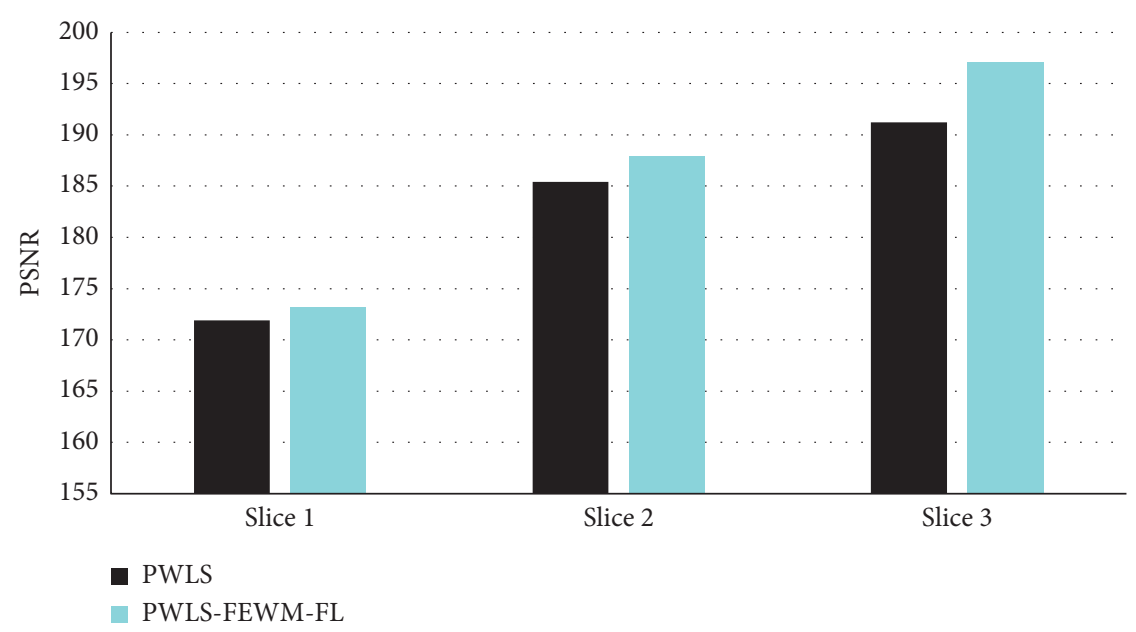

FIGURE 5: Contrast of the PSNR value of noise reduction algorithm between slices.

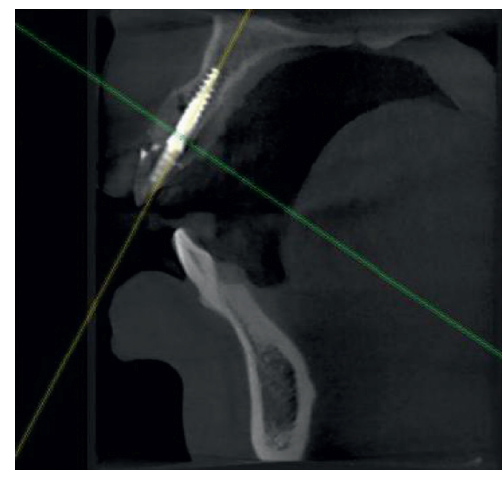

(a)

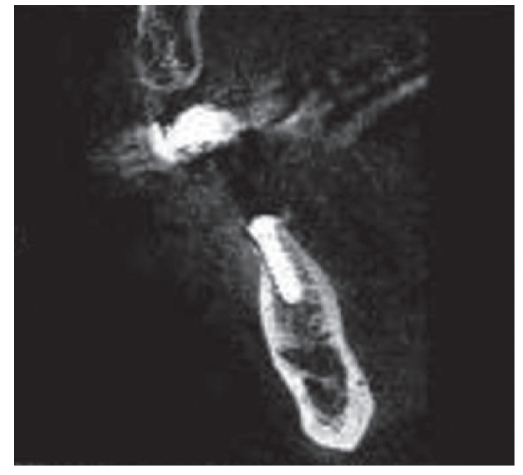

(b)

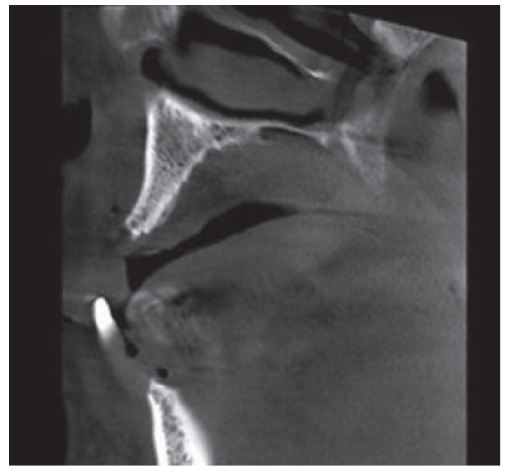

(c)

FIGURE 6: CBCT images: (a) lacked filled; (b) well filled; (c) over filled.

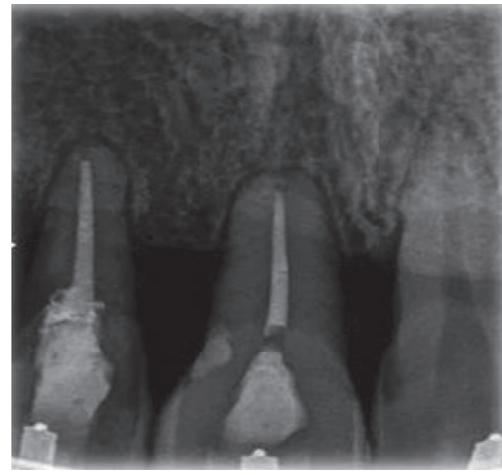

(a)

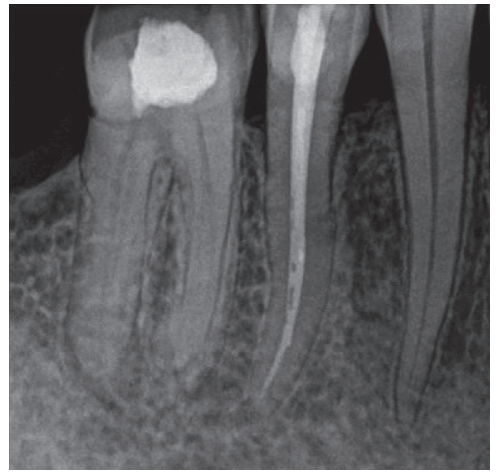

(b)

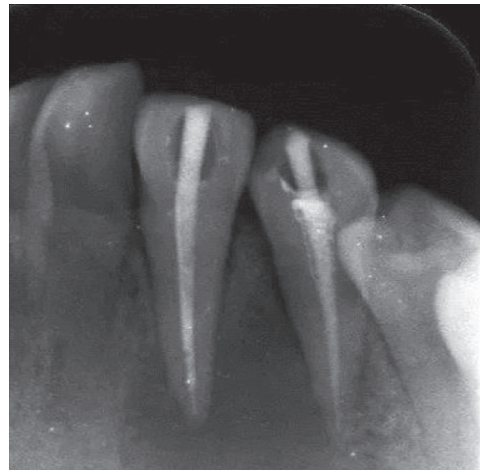

(c)

FIGURE 7: DPF images: (a) well filled without gap; (b) lacked filled; (c) well filled with gap.

in Figure 10. Results of CBCT imaging technology suggested that 108 patients showed satisfaction and 126 patients showed dissatisfaction on the root canal filling quality.
When DPF was utilized to evaluate root canal quality, 155 patients showed satisfaction and 79 patients showed dissatisfaction. For the evaluated results of root canal filling 


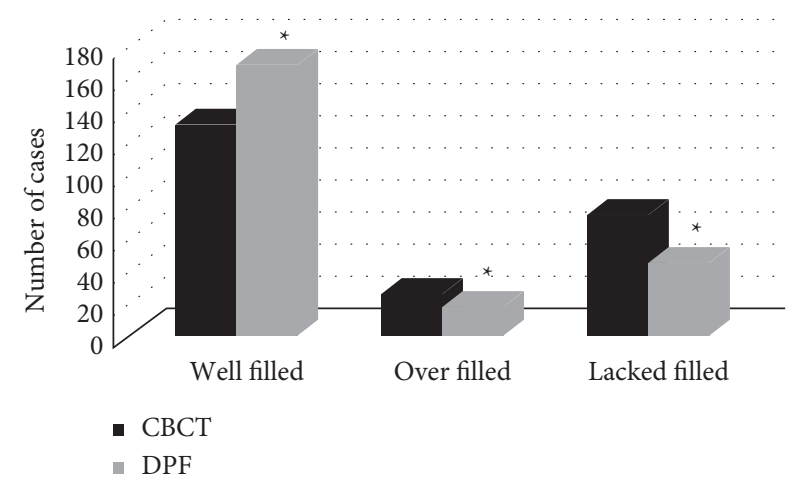

Figure 8: Contrast of root canal filling length evaluation results $\left({ }^{*}\right.$ indicated that the difference was considerable relative to $\mathrm{CBCT}$, $P<0.05)$.

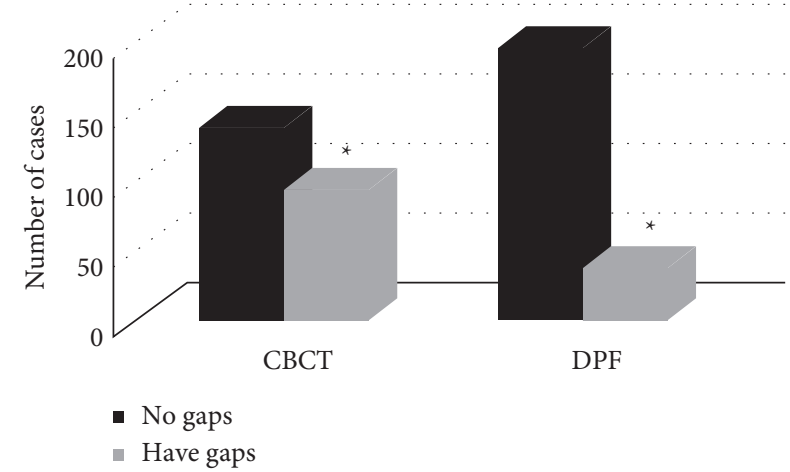

Figure 9: Contrast of evaluation results of root canal closeness $\left({ }^{*}\right.$ indicated that the difference was considerable relative to $\mathrm{CBCT}$, $P<0.05)$.

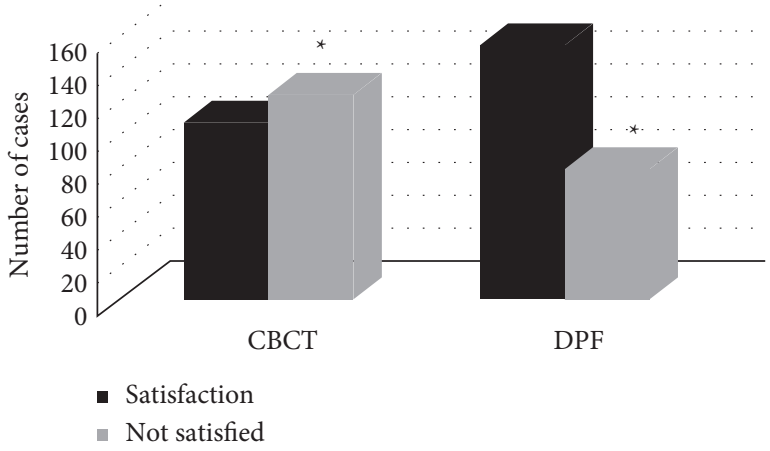

Figure 10: Contrast of evaluation results of root canal filling quality $\left({ }^{*}\right.$ indicated that the difference was considerable relative to CBCT, $P<0.05)$.

quality, differences between CBCT technology and DPF were greatly considerable $(P<0.05)$.

\section{Discussion}

X-ray CT technology has been widely utilized in medical clinical diagnosis. There are many studies on the improvement of the built-in algorithm of CT imaging and the evaluation of imaging quality. The main purpose is to obtain ideal image pathological characteristics and reduce the radiation damage of X-ray beam [13]. Adopting CBCT scanning to obtain $3 \mathrm{D}$ projection data are an effective means of radiation dose control, but it will affect the imaging quality to a certain extent. To obtain a lower noise level in the reconstructed image and maintain better detail information and image resolution, the penalty-weighted least squares noise reduction algorithm and the fuzzy entropy mean filter algorithm were combined. Based on which, the CBCT imaging technology under INR algorithm was proposed to process medical CBCT images. The results showed that the CBCT images under the INR algorithm had achieved relatively ideal noise reduction effects within and between slices, which was consistent with the research results of $\mathrm{Hu}$ et al. [14].

X-ray can be utilized to diagnose the status of affected teeth in the process of RCT, so as to have a clear understanding of the morphology and length of the root canal in affected teeth, judge the inflammation range, and evaluate the filling effect of the root canal. In clinic, DPF is mostly utilized, but the tooth structure is $3 \mathrm{D}$, so the DPF of $2 \mathrm{D}$ images has certain limitations in the judgment of diseases. High-resolution 3D images overcome limitations such as the superposition and anatomical noise of $2 \mathrm{D}$ conventional imaging, making $\mathrm{CBCT}$ an increasingly popular imaging modality in many dental applications. CBCT technology can observe the target object in the 3D direction without overlapping images. The subsequent $3 \mathrm{D}$ reconstruction by software can better show the real tooth structure and morphological characteristics. Leonardi et al. [15] showed that the accuracy of CBCT in the diagnosis of dental pulp disease was 0.96, which was better than that of DPF (0.72). DPF and CBCT were utilized to evaluate the efficacy of RCT, and it was found that the evaluation results in the root canal filling length, the root canal closeness, and the root canal filling quality were evidently different $(P<0.05)$. It was consistent with the research results of Lima et al. [16]. In this study, it was found that, in contrast with DPF, CBCT had a more significant advantage in detecting gap in root filling, which was also confirmed by many previous studies. In addition, relevant anatomical and histological studies also showed that gap observed by CBCT was real [17]. The main reason was that $\mathrm{DPF}$, as a $2 \mathrm{D}$ image technology, inevitably produced image overlapping and gap was difficult to be found. However, some DPF images with root filling gap were not found in CBCT images, which may be due to the limited image resolution of CBCT in small transmission area.

\section{Conclusion}

INR algorithm was applied to improve the image quality of CBCT, which was then adopted in the curative effect analysis of RCT for dental pulp patients. It was found that the CBCT imaging technology based on the INR algorithm had achieved ideal noise reduction effects within and between slices, and the image information was retained better. СВCT imaging technology and DPF had considerably differences in 
the evaluation results of root canal filling length, root canal closeness, and root canal filling quality in patients with dental pulp disease $(P<0.05)$, and CBCT images were superior to those of DPF. In short, the results can provide a reference for the imaging diagnosis of dental pulp disease and RCT research.

However, the research still has certain shortcomings. For example, the sample size is limited and only the short-term efficacy is evaluated. In the future, in the adoption research of CBCT based on INR algorithm in different RCTs, it will further expand the sample size and provide more reliable data support for the research results. Moreover, the longterm effect of different RCTs evaluated by CBCT based on INR algorithm should be discussed, which will create more feasibility for the popularization and adoption of such kind of CT.

\section{Data Availability}

The data used to support the findings of this study are available from the corresponding author upon request.

\section{Conflicts of Interest}

The authors declare no conflicts of interest.

\section{References}

[1] H. Jafarzadeh, Z. Mohammadi, S. Shalavi, and J.-I. Kinoshita, "Unusual root canal irrigation solutions," The Journal of Contemporary Dental Practice, vol. 18, no. 5, pp. 415-420, 2017.

[2] N. Chandler and D. Chellappa, "Lubrication during root canal treatment," Australian Endodontic Journal, vol. 45, no. 1, pp. 106-110, 2019.

[3] P. Neelakantan, M. Romero, J. Vera et al., "Biofilms in endodontics-current status and future directions," International Journal of Molecular Sciences, vol. 18, no. 8, p. 1748, 2017.

[4] C. Kruse, R. Spin-Neto, J. Reibel, A. Wenzel, and L.-L. Kirkevang, "Diagnostic validity of periapical radiography and CBCT for assessing periapical lesions that persist after endodontic surgery," Dentomaxillofacial Radiology, vol. 46, no. 7, Article ID 20170210, 2017.

[5] H. Gaêta-Araujo, E. H. L. Nascimento, D. M. Brasil, A. F. Gomes, D. Q. Freitas, and C. De Oliveira-Santos, "Detection of simulated periapical lesion in intraoral digital radiography with different brightness and contrast," Eur Endod J, vol. 4, no. 3, pp. 133-138, 2019.

[6] Â. G. Deliga Schröder, F. H. Westphalen, J. C. Schröder, $\hat{A}$. Fernandes, and V. P. D. Westphalen, "Accuracy of digital periapical radiography and cone-beam computed tomography for diagnosis of natural and simulated external root resorption," Journal of Endodontics, vol. 44, no. 7, pp. 1151-1158, 2018.

[7] F. Yılmaz, K. Kamburoğlu, and B. Şenel, "Endodontic working length measurement using cone-beam computed tomographic images obtained at different voxel sizes and field of views, periapical radiography, and apex locator: a comparative ex vivo study," Journal of Endodontics, vol. 43, no. 1, pp. 152-156, 2017.
[8] D. Song, L. Zhang, W. Zhou et al., "Comparing cone-beam computed tomography with periapical radiography for assessing root canal obturation in vivo using microsurgical findings as validation," Dentomaxillofacial Radiology, vol. 46, no. 5, Article ID 20160463, 2017.

[9] M. B. McGuigan, H. F. Duncan, and K. Horner, "An analysis of effective dose optimization and its impact on image quality and diagnostic efficacy relating to dental cone beam computed tomography (CBCT)," Swiss dental journal, vol. 128, no. 4, pp. 297-316, 2018.

[10] X. Zheng, S. Ravishankar, Y. Long, and J. A. Fessler, "PWLSULTRA: an efficient clustering and learning-based approach for low-dose 3D CT image reconstruction," IEEE Transactions on Medical Imaging, vol. 37, no. 6, pp. 1498-1510, 2018.

[11] Z. Bian, D. Zeng, Z. Zhang et al., "Low-dose dynamic myocardial perfusion CT imaging using a motion adaptive sparsity prior," Medical Physics, vol. 44, no. 9, pp. e188-e201, 2017.

[12] J. Lin, H. Zhang, J. Huang et al., "Iterative reconstruction for low dose dual energy CT using information-divergence constrained spectral redundancy information," Journal of X-Ray Science and Technology, vol. 26, no. 2, pp. 311-330, 2018.

[13] J.-P. Grunz, A. S. Kunz, C. H. Gietzen et al., “3D cone-beam $\mathrm{CT}$ of the ankle using a novel twin robotic X-ray system: assessment of image quality and radiation dose," European Journal of Radiology, vol. 119, Article ID 108659, 2019.

[14] Z. Hu, J. Gao, N. Zhang et al., "An improved statistical iterative algorithm for sparse-view and limited-angle CT image reconstruction," Scientific Reports, vol. 7, no. 1, Article ID 10747, 2017.

[15] K. Leonardi Dutra, L. Haas, A. L. Porporatti et al., "Diagnostic accuracy of cone-beam computed tomography and conventional radiography on apical periodontitis: a systematic review and meta-analysis," Journal of Endodontics, vol. 42, no. 3, pp. 356-364, 2016, PMID: 26902914.

[16] T. Lima, T. Gamba, A. Zaia, and A. Soares, "Evaluation of cone beam computed tomography and periapical radiography in the diagnosis of root resorption," Australian Dental Journal, vol. 61, no. 4, pp. 425-431, 2016.

[17] J. N. R. Martins, A. Kishen, D. Marques et al., "Preferred reporting items for epidemiologic cross-sectional studies on root and root canal anatomy using cone-beam computed tomographic technology: a systematized assessment," Journal of Endodontics, vol. 46, no. 7, pp. 915-935, 2020. 